\title{
ON THE FACTORIZATION OF $A_{p}$ WEIGHTS
}

\author{
LI XING-MIN
}

(Communicated by J. Marshall Ash)

ABstract. It is shown that if $(u, v) \in A_{p}$ then there exist $\left(u_{1}, v_{1}\right) \in A_{1}$ and $\left(u_{2}, v_{2}\right) \in A_{1}$ such that $u=u_{1}^{p^{\prime}} v_{2}^{-p}, v=v_{1}^{p^{\prime}} u_{2}^{-p}$.

\section{INTRODUCTION}

Let $M$ denote the Hardy-Littlewood maximal operator in $R^{n}$,

$$
M f(x)=\sup _{x \in Q} \frac{1}{|Q|} \int_{Q}|f|,
$$

where $Q$ is always a cube in $R^{n}$ and $|\cdot|$ denotes Lebesgue measure. Let $w(x)$, $u(x)$, and $v(x)$ be nonnegative measurable functions on $R^{n}$, and recall that [1]

$$
\begin{array}{ll}
W \in A_{1} & \text { if and only if } \quad M w(x) \leq C w(x) \text { a.e. } x \in R^{n} ; \\
w \in A_{p} & \text { if and only if } \quad\left(\int_{Q} w\right)\left(\int_{Q} w^{1-p^{\prime}}\right)^{p-1} \leq C|Q|^{p} \\
(u, v) \in A_{1} & \text { if and only if } \quad M u(x) \leq C v(x) \text { a.e. } x \in R^{n} ; \\
(u, v) \in A_{p} & \text { if and only if } \quad\left(\int_{Q} u\right)\left(\int_{Q} v^{1-p^{\prime}}\right)^{p-1} \leq C|Q|^{p} \\
(u, v) \in S_{p} & \text { if and only if } \quad \int|M f|^{p} u \leq C \int|f|^{p} v .
\end{array}
$$

Throughout $C$ denotes a constant and may vary from line to line, $1<p<\infty$ (unless otherwise noted), and $\frac{1}{p}+\frac{1}{p^{\prime}}=1$.

If there exist $C_{1}>0$ and $C_{2}>0$ such that $C_{1} u \leq v \leq C_{2} u$ a.e. $x \in R^{n}$, then $u \sim v$.

In [2] Jones showed that $w \in A_{p}$ if and only if there exist weights $w_{1}$, $w_{2} \in A_{1}$ with $w=w_{1} w_{2}^{1-p}$. In this note we consider the factorization of the general $A_{p}$ weights. Our results are as follows.

Theorem 1. Let $(u, v) \in A_{p}$ and $0<\delta<1$. Then there exist $\left(u_{1}, v_{1}\right)$ and $\left(u_{2}, v_{2}\right) \in A_{1}$ such that

$$
u^{\delta}=u_{1} v_{2}^{1-p}, \quad v^{\delta}=v_{1} u_{2}^{1-p} .
$$

Received by the editors November 12, 1991 and, in revised form, November 1, 1992.

1991 Mathematics Subject Classification. Primary 42B25. 
Moreover, if $u, v \in L_{\mathrm{loc}}^{1}\left(R^{n}\right)$ and $0<u, v<\infty$ a.e. $x \in R^{n}$, then $u \sim v$ if and only if $u_{1} \sim v_{1}$ and $u_{2} \sim v_{2}$.

Theorem 2. There exist $\left(u_{1}, v_{1}\right) \in A_{1}$ and $\left(u_{2}, v_{2}\right) \in A_{1}$ such that for any $0<\delta<1$

$$
(u, v)=\left(\left(u_{1} v_{2}^{1-p}\right)^{1 / \delta},\left(v_{1} u_{2}^{1-p}\right)^{1 / \delta}\right) \notin A_{p} .
$$

This shows that the converse of Theorem 1 is false.

Corollary 1. If $(u, v) \in A_{p}$, then there exist $\left(u_{1}, v_{1}\right)$ and $\left(u_{2}, v_{2}\right) \in A_{1}$ such that

$$
u=u_{1}^{p^{\prime}} v_{2}^{-p}, \quad v=v_{1}^{p^{\prime}} v_{2}^{-p} .
$$

Proof. Using Theorem 1 with $\delta=1 / p^{\prime}$, we get the result.

Corollary 2. If $w \in A_{p}$, then there exist $w_{1}, w_{2} \in A_{1}$ such that $w=w_{1} w_{2}^{1-p}$. Proof. We may assume that $0<w<\infty$ a.e. so that $w \in L_{\mathrm{loc}}^{1}\left(R^{n}\right)$. Since $w \in A_{p}$, there exists $\tau>1$ such that $w^{\tau} \in A_{p}$ [1]. Let $\delta=1 / \tau$. By Theorem 1 there exist $\left(u_{1}, v_{1}\right),\left(u_{2}, v_{2}\right) \in A_{1}$ with $u_{1} \sim v_{1}, u_{2} \sim v_{2}$, and therefore $u_{1}, u_{2} \in A_{1}$, such that $\left(w^{\tau}\right)^{1 / \tau}=u_{1} v_{2}^{1-p}$, i.e., $w=u_{1} v_{2}^{1-p}$. Let $u_{1}=w_{1}$, $v_{2}=w_{2}$; we get $w=w_{1} w_{2}^{1-p}$ with $w_{1}, w_{2} \in A_{1}$.

\section{Proof OF THE THEOREMS}

We need the following lemmas.

Lemma 1 [3]. If $(u, v) \in A_{p}$, and $0<\delta<1$, then $\left(u^{\delta}, v^{\delta}\right) \in S_{p}$.

Lemma 2 [3]. Assume that $(u, v) \in S_{p}$ and $\left(v^{1-p^{\prime}}, u^{1-p^{\prime}}\right) \in S_{p^{\prime}}$. Then there are functions $w_{j} \geq 0$ such that

$$
u^{1 / p} M w_{j} \leq C_{j} w_{j} v^{1 / p}, \quad j=1,2,
$$

and

$$
u^{1 / p} v^{1 / p^{\prime}}=w_{1} w_{2}^{1-p}
$$

Lemma 3. If $(u, v) \in A_{p} \quad(1 \leq p<\infty)$ and $u, v \in L_{\mathrm{loc}}^{1}\left(R^{n}\right)$, then $u(x) \leq$ $C v(x)$ a.e. $x \in R^{n}$.

Proof. For $p=1$ it is obvious. For $1<p<\infty$, since $(u, v) \in A_{p}$ if and only if [1]

$$
\left(1 /|Q| \int_{Q} f\right)^{p}\left(\int_{Q} u\right) \leq C \int_{Q} f^{p} v
$$

for any $f \geq 0, Q \subset R^{n}$, let $f \equiv 1$. Then $\int_{Q} u \leq C \int_{Q} v$, i.e.,

$$
1 /|Q| \int_{Q} u \leq C 1 /|Q| \int_{Q} v \text {. }
$$

Let $|Q| \rightarrow 0$. Since $u, v \in L_{\text {loc }}^{1}\left(R^{n}\right)$ we get

$$
u(x) \leq C v(x) \text { a.e. } x \in R^{n} \text {. }
$$

Lemma 4. If $(u, v) \in S_{p}$ and $\left(v^{1-p^{\prime}}, u^{1-p^{\prime}}\right) \in S_{p^{\prime}}$, then there exist $\left(u_{j}, v_{j}\right) \in$ $A_{1}, j=1,2$, such that

$$
u=u_{1} v_{2}^{1-p}, \quad v=v_{1} u_{2}^{1-p} .
$$


Proof. Let $u_{j}=w_{j}$ and $v_{j}=w_{j} v^{1 / p} u^{-1 / p}$ by Lemma 2. Then $\left(u_{j}, v_{j}\right) \in A_{1}$, $j=1,2$.

Since $u^{1 / p} v^{1 / p^{\prime}}=w_{1} w_{2}^{1-p}$, we get

$$
u=w_{1}^{p} w_{2}^{p(1-p)} v^{1-p}, \quad v=w_{1}^{p^{\prime}} w_{2}^{-p} u^{1-p^{\prime}} .
$$

Thus,

$$
\begin{aligned}
u_{1} v_{2}^{1-p} & =w_{1}\left(w_{2} v^{1 / p} u^{-1 / p}\right)^{1-p} \\
& =w_{1} w_{2}^{1-p} v^{-1 / p^{\prime}}\left(w_{1}^{p} w_{2}^{p(1-p)} v^{1-p}\right)^{1 / p^{\prime}}=w_{1}^{p} w_{2}^{p(1-p)} v^{1-p}=u
\end{aligned}
$$

and

$$
\begin{aligned}
v_{1} u_{2}^{1-p} & =\left(w_{1} v^{1 / p} u^{-1 / p}\right) w_{2}^{1-p} \\
& =w_{1} u^{-1 / p}\left(w_{1}^{p^{\prime}} w_{2}^{-p} u^{1-p^{\prime}}\right)^{1 / p} w_{2}^{1-p}=w_{1}^{p^{\prime}} w_{2}^{-p} u^{1-p^{\prime}}=v .
\end{aligned}
$$

Proof of Theorem 1. Since $(u, v) \in A_{p}$ if and only if $\left(v^{1-p^{\prime}}, u^{1-p^{\prime}}\right) \in A_{p^{\prime}}$. Lemma 1 gives $\left(u^{\delta}, v^{\delta}\right) \in S_{p}$ and $\left(v^{\delta\left(1-p^{\prime}\right)}, u^{\delta\left(1-p^{\prime}\right)}\right) \in S_{p^{\prime}}$. From Lemma 4 we get $\left(u_{j}, v_{j}\right) \in A_{1}, j=1,2$, such that

$$
u^{\delta}=u_{1} v_{2}^{1-p}, \quad v^{\delta}=v_{1} u_{2}^{1-p} .
$$

For $u, v \in L_{\text {loc }}^{1}\left(R^{n}\right)$ and $0<u, v<\infty$ a.e. if $u_{1} \sim v_{1}, u_{2} \sim v_{2}$, then $u_{1} v_{2}^{1-p} \sim v_{1} u_{2}^{1-p}$. Hence $u^{\delta} \sim v^{\delta}$, i.e., $u \sim v$.

Conversely, if $u \sim v$, then there exist $C_{1}>0, C_{2}>0$ such that

$$
C_{1} v_{1} u_{2}^{1-p} \leq u_{1} v_{2}^{1-p} \leq C_{2} v_{1} u_{2}^{1-p} .
$$

From the left inequality, we have

$$
\left(v_{2} / u_{2}\right)^{p-1} \leq C u_{1} / v_{1} .
$$

Note that $u_{j} \leq C v_{j}, j=1,2$. We get $u_{2} \sim v_{2}$ and also $u_{1} \sim v_{1}$.

Proof of Theorem 2. Let $u_{1}=v_{1} \equiv 1, u_{2}=\varphi(x)=\chi_{[0,1]}$, and $v_{2}=M \varphi$. Then $\left(u_{j}, v_{j}\right) \in A_{1}, j=1,2$, but $\left(u_{1} v_{2}^{1-p}, v_{1} u_{2}^{1-p}\right)=\left((M \varphi)^{1-p}, \varphi^{1-p}\right) \notin S_{p}$. In fact, let $f=\varphi=\chi_{[0,1]}$. Then we have

$$
\int(M f)^{p}(M \varphi)^{1-p}=\int M \varphi=+\infty,
$$

but $\int|f|^{p} \varphi^{1-p}=\int \varphi=1$. 1 .

From Lemma $1,(u, v)=\left(\left(u_{1} v_{2}^{1-p}\right)^{1 / \delta},\left(v_{1} u_{2}^{1-p}\right)^{1 / \delta}\right) \notin A_{p}$ for any $0<\delta<$

\section{REFERENCES}

1. J. Garcia-Cuerva and J. L. Rubio de Francia, Weighted norm inequalities and related topics, North-Holland, Amsterdam, 1985.

2. P. Jones, Factorization of $A_{p}$ weights, Ann. of Math. (2) 111 (1980), 511-530.

3. C. J. Neugebauer, Inserting $A_{p}$-weights, Proc. Amer. Math. Soc. 87 (1983), 644-648.

Department of Mathematics, Qu Fu Normal University, Shan Dong Province, 273165 People's Republic of China 